\title{
AVALIAÇÃO DA DEMANDA DE ÁGUA DEVIDO A DIFERENTES PRESSÕES EM BACIAS SANITÁRIAS COM CAIXA ACOPLADA EM EDIFÍCIOS RESIDENCIAIS
}

\author{
Gelcimara Bento da Silva ${ }^{1}$, Mario Tachini² e Abrahão Bernardo Rohden ${ }^{3}$
}

\begin{abstract}
Resumo: $O$ estudo da pressão em mecanismos de acionamento (MAC) de reparo para bacias sanitárias pode proporcionar uma diminuição considerável no consumo de água em unidades residenciais, tornando o sistema mais econômico e sustentável. As bacias sanitárias são um dos aparelhos responsáveis pelo maior consumo de água e a alta pressão é um fator agravante. Esse consumo se deve a perda de água que pode acontecer quando ocorre o reenchimento da caixa, enquanto o botão de acionamento está sendo pressionado. O presente trabalho teve como objetivo estudar o consumo de água em bacias sanitárias com caixa acoplada utilizando três tipos de modelos de MAC para reparo que foram submetidos a diferentes pressões. Foi acionada a descarga durante dois tempos, 1 e $2 \mathrm{~s}$, sendo que para cada tempo se adotou quatro diferentes valores de pressão, 10, 20, 30 e 36 m.c.a., repetindo-se o procedimento dez vezes para cada combinação e para cada MAC. O MAC 3 foi o que apresentou a maior variação média de consumo de água que ficou entre 6,57 L e $7,55 \mathrm{~L}$. O aumento do consumo de água pelo MAC gerou um impacto econômico $R \$ 5.439,28$ na fatura de água e esgoto anual em um edifício multifamiliar, com 48 apartamentos distribuídos em 12 pavimentos.
\end{abstract}

Palavras-chave: Bacia sanitária. Caixa acoplada. Demanda de água. Desperdício.

\section{Introdução}

Com a necessidade de economia de água e a preservação dos recursos naturais, buscam-se alternativas para mudanças nos hábitos da população e melhorias nos equipamentos hidrossanitários. No mercado existem várias alternativas tecnológicas com esse propósito e algumas delas consistem no uso de equipamentos economizadores de água, como por exemplo as bacias sanitárias com caixa acoplada, lavatórios e chuveiros com volume de escoamento reduzido, torneiras com arejadores que concentram a vazão da água.

Em uma edificação as bacias sanitárias consomem uma quantidade de água considerável com relação aos outros equipamentos sanitários, devido a diferentes fatores, como por exemplo, instalação inclinada do aparelho sanitário, falta de manutenção adequada e devido a pressões elevadas na entrada dos aparelhos. A alta pressão em tubulações de água fria é uma das principais razões de aumento no consumo de água nos aparelhos sanitários. De acordo com Cabral et al. (2016), nas caixas de descarga de bacias sanitárias, pode ocorrer uma perda da água quando a água entra na caixa acoplada durante o acionamento, devido à grande pressão que chega na tubulação.

Segundo Oliveira (2005), no Brasil, em cidades de porte médio, o consumo per capita nas residências chega a 200 L/hab./dia, e em bairros mais nobres o consumo pode se elevar até $400 \mathrm{~L} / \mathrm{hab}$./dia. Já em cidades de pequeno porte, o consumo pode se reduzir para $120 \mathrm{~L} / \mathrm{hab} . / \mathrm{dia}$. De acordo com Annecchini (2005), o consumo de água em uma residência pode ser influenciado por diversos fatores, tais como o clima, o número de habitantes, aspectos culturais e até mesmo a forma de gerenciamento do sistema de abastecimento de água.

Tomaz (2009) estima que em uma residência, uma pessoa aciona a descarga da bacia sanitária em média 5 vezes por dia. Este valor ainda pode variar entre 1 descarga para mais ou para menos. Por sua vez a American Water Works Association (AWWA, 1999, apud UCHIDA; OLIVEIRA, 2006) sugere que em uma residência a quantidade de água consumida por uma bacia sanitária pode corresponder a até $26,7 \%$ do consumo total.

$\overline{{ }^{1} E-m a i l: ~ g e l c i m a r a @ h o t m a i l . c o m ~}$

1E-mail: mtachini@furb.br

${ }^{1} \mathrm{E}$-mail: arohden@furb.br

FURB - Universidade Regional de Blumenau, Departamento de engenharia civil. CAMPUS II Complexo Tecnológico Rua São Paulo, 3250. Itoupava Seca - 89030-000 Blumenau - SC 


\section{REA - Revista de estudos ambientais (Online) v.21, n. 2, p.48-57, jul./dez. 2019}

A propósito, Fracasso (2017) realizou um estudo no município de Lages/SC também voltado ao consumo de água em uma residência. Os resultados da pesquisa, apontados na Tabela 1, confirmam que neste caso o maior consumo de água em uma residência se deve às bacias sanitárias.
É muito importante observar que na pesquisa realizada no município de Blumenau/SC, um dos maiores consumos de água nas residências também se deve à bacia sanitária (Tabela 2).

Tabela 1 - Consumo de água em uma residência

\begin{tabular}{lc}
\hline \multicolumn{1}{c}{ Aparelhos } & Consumo (\%) \\
\hline Descarga da bacia sanitária & 41 \\
Chuveiro & 37 \\
Pia (cozinha) & 6 \\
Bebida & 5 \\
Lavação de roupas & 4 \\
Limpeza da casa & 3 \\
Irrigação de jardins & 3 \\
Lavação de carros & 1 \\
\hline Fracasso (2017)
\end{tabular}

Fonte: Fracasso (2017)

Tabela 2 - Percentagem média dos usos finais de água em residências de Blumenau/SC

\begin{tabular}{lcccccc}
\hline \multirow{2}{*}{\multicolumn{1}{c}{ Aparelho }} & \multicolumn{6}{c}{ Percentagem média dos usos finais de água (\%) } \\
\cline { 2 - 7 } & RB & R2 & R3 & R4 & R5 & R6 \\
\hline Torneira do banheiro & 7,3 & 4,7 & 5,7 & 11,4 & 3,4 & 6,0 \\
Bacia sanitária & 21,6 & 9,9 & 37,2 & 25,7 & 19,3 & 17,3 \\
Chuveiro & 17,5 & 29,6 & 29,3 & 11,4 & 32,8 & 53,5 \\
Torneira da cozinha & 14,4 & 8,7 & 9,0 & 19,4 & 10,4 & 11,6 \\
Mangueira de jardim & 13,5 & - & - & - & 5,5 & - \\
Torneira do tanque & 7,2 & 1,4 & 4,9 & 2,9 & 0,5 & 2,7 \\
Máquina de lavar roupa & 18,5 & 30,7 & 13,2 & 29,0 & 27,9 & 8,6 \\
Ingestão (filtro) & - & 1,0 & 0,6 & 0,3 & 0,2 & 0,3 \\
Torneira externa & - & 13,9 & - & - & - & - \\
Total & 100,0 & 100,0 & 100,0 & 100,0 & 100,0 & 100,0 \\
\hline
\end{tabular}

Onde: RB é a residência base e R2 a R6 são 5 residências onde foram feitas medições. Fonte: Coelho (2008)

A NBR 15097-1 (ABNT, 2017) define bacias sanitárias com caixa acoplada, como sendo uma bacia que possua uma caixa de descarga para uso específico, na qual a bacia e o seu reservatório devem ser comercializados em conjunto. De acordo com a NBR 15491 (ABNT, 2010) as bacias sanitárias comercializadas no Brasil devem possuir um volume de descarga reduzido de 6,8 L, controláveis por mecanismos (MAC), instalados no interior das caixas acopladas das bacias sanitárias.

Atualmente, o mercado possui uma grande variedade de bacias sanitárias, das quais pode-se citar a convencional (comercializada sem o aparelho de descarga correspondente), a com caixa acoplada e a integrada (formando um só corpo). Neste trabalho estudou-se a bacia sanitária com caixa acoplada, que consome aproximadamente $6 \quad \mathrm{~L}$ de água por acionamento.

Para Lombardi (2012) as bacias sanitárias com volume de descarga reduzido (VDR) começaram a ser comercializadas no Brasil em 1999, o que proporcionou uma redução considerável no consumo de água e diminuiu de forma significativa a emissão de efluentes. A Figura 1 apresenta um modelo bastante comum de bacia sanitária com caixa acoplada disponível no mercado. 


\section{REA - Revista de estudos ambientais (Online) v.21, n. 2, p.48-57, jul./dez. 2019}

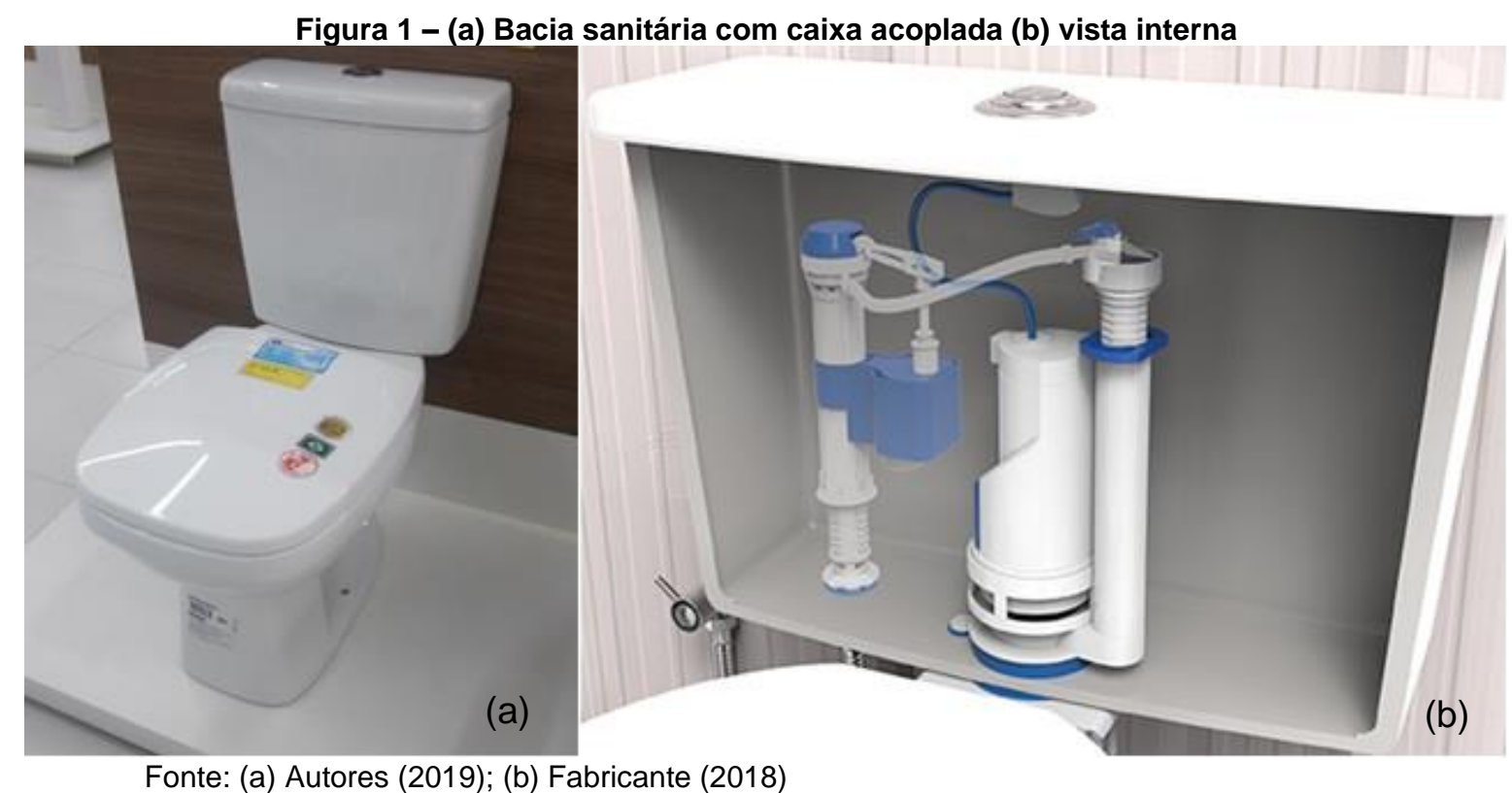

Para Reali et al. (2002), as bacias sanitárias com caixa acoplada são muito empregadas ao invés de válvulas de descarga, pois apresentam algumas vantagens, como diâmetros de tubulações menores, inexistência de problemas de oscilações nas pressões e ainda economia de construção.

Cabral et al. (2016) destacam que nas caixas de descarga convencionais, pode ocorrer perda de água na hora do reenchimento, ou seja, se o volume da caixa cheia é de $6 \mathrm{~L}$, ao final o volume efetivo pode variar para cerca de $7 \mathrm{~L}$ ou mais por descarga. Portanto, existe uma perda de água, que depende da pressão que chega da rede, e se dá até que o obturador vede novamente.

Graças aos recentes estudos sobre o consumo de água em bacias sanitárias, novas tecnologias tornam os sistemas de descarga mais econômicos. Com estes avanços, as bacias sanitárias convencionais foram substituídas por modelos mais inovadores e economizadores, que se mostram muito eficientes na redução do consumo e economia de água (UCHIDA; OLIVEIRA, 2006).

O objetivo do presente trabalho foi avaliar o consumo de água de diferentes MACs de bacias sanitárias disponíveis no mercado em diferentes condições de pressão e tempos de acionamento de válvula, buscando assim entender a influência destas variáveis no consumo de água. Também se realizou uma avaliação do impacto dos diferentes consumos de água no custo de água e esgoto de um edifício de 12 pavimentos.

\section{Metodologia}

Os ensaios foram realizados no laboratório de hidráulica da Universidade Regional de Blumenau (FURB)/SC. O sistema é abastecido de água fria a partir do barrilete que abastece os vários aparelhos sanitários dispostos no laboratório. A Figura 2 (a) e 2 (b) mostram o protótipo utilizado no estudo. $O$ sistema de abastecimento passa inicialmente por um conjunto motobomba com capacidade máxima de bombeamento em serviço de 36 m.c.a., seguido por um sistema de redução de pressão. A pressão da água que passa nas colunas de distribuição foi regulada com o auxílio de uma válvula redutora de pressão (VRP), não pilotada. No sistema foi instalado um manômetro para monitoramento da pressão de entrada na caixa acoplada. Os tubos e conexões empregados na instalação são de PVC com diâmetro nominal 20 mm $(1 / 2 ")$.

Nos ensaios foram testados três MACs completos de diferentes marcas, porém todos puderam ser adaptados à bacia sanitária (Figura 3). 


\section{REA - Revista de estudos ambientais (Online) v.21, n. 2, p.48-57, jul./dez. 2019}

Figura 2 - (a) Vista geral do protótipo e (b)
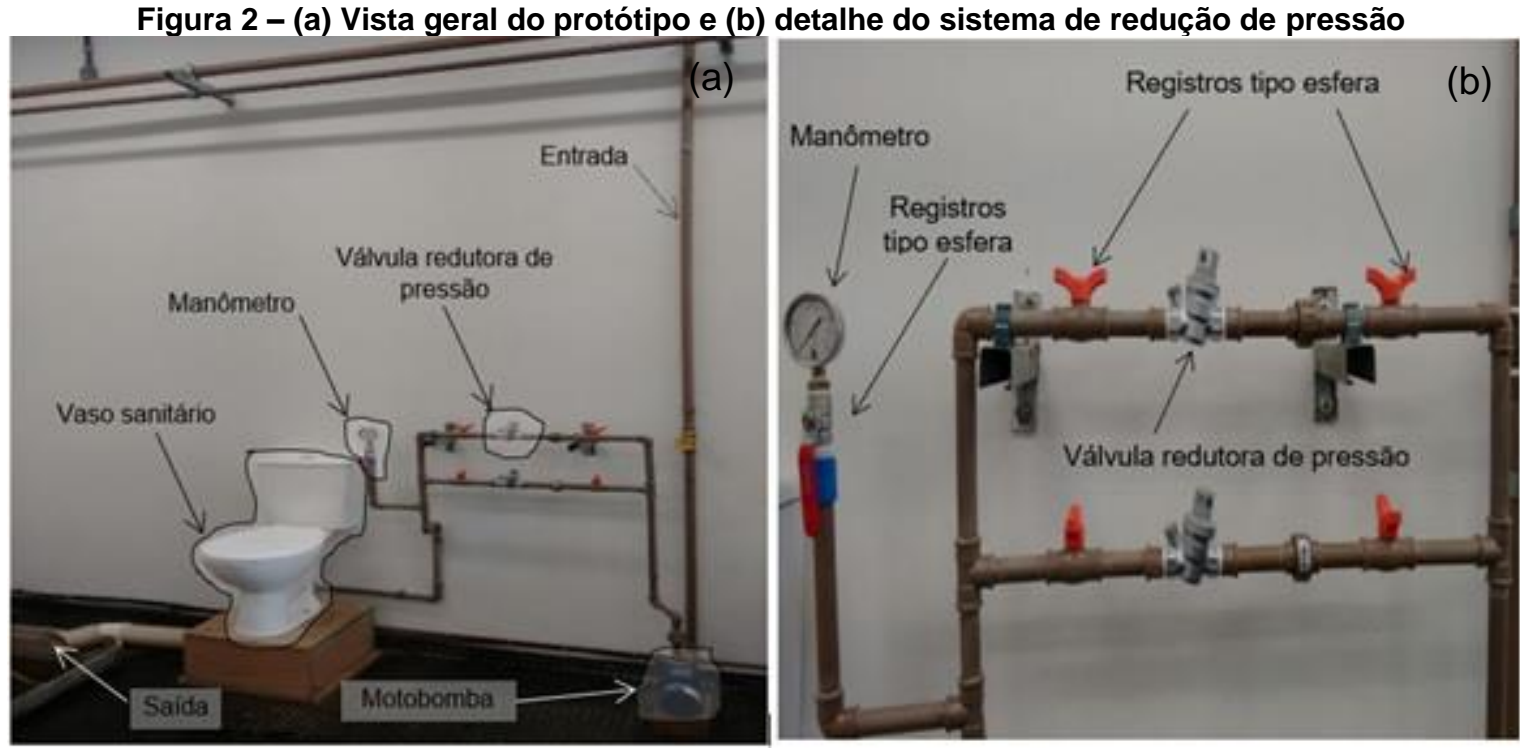

Fonte: Autores (2019)

Figura 3 - Vista geral dos MACs ensaiados MAC1 (a), MAC2 (b) e MAC (3)

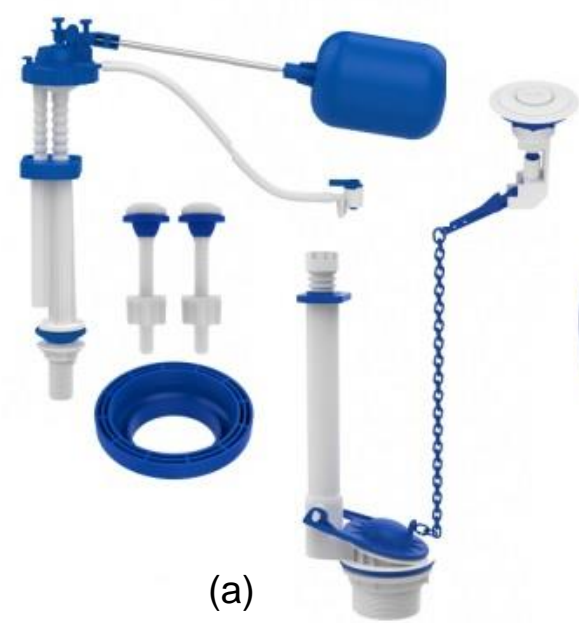

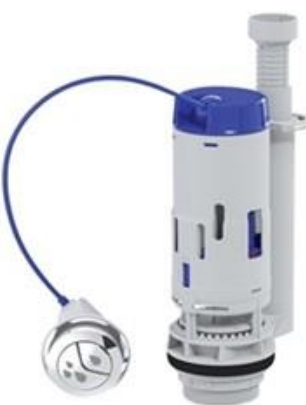

(b)
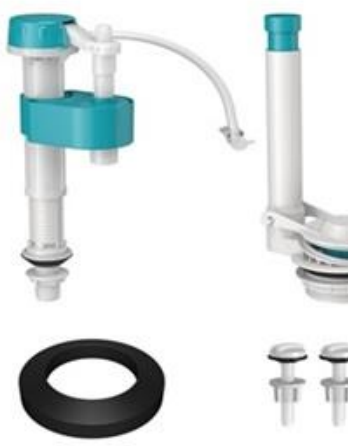

(c)

Adaptado dos fabricantes (2018)

Para cada MAC instalado o volume da caixa de descarga inicial foi aferido com o auxílio de um recipiente volumétrico (béquer), após o acionamento da descarga com uma pressão estática de 2,08 m.c.a., existente na entrada da bacia sanitária. Com a caixa de descarga da bacia sanitária em pressão estática foi realizada a regulagem da válvula redutora de pressão. $\mathrm{Na}$ Figura 4 é apresentado o fluxograma com as variáveis estudadas no trabalho. Para cada MAC testado realizaram-se quatro testes nas condições de 10, 20, 30 e 36 m.c.a. As pressões adotadas foram delimitadas em função das pressões utilizadas em instalações hidráulicas prediais. Os ensaios foram realizados para acionamentos de descarga de 1 e $2 \mathrm{~s}$. Após o acionamento da descarga, um recipiente volumétrico foi posicionado na saída da bacia sanitária, possibilitando a coleta e quantificação volumétrica da água. Para cada combinação foram realizadas 10 determinações (repetições). A água coletada na saída da canalização foi transferida para uma proveta com a qual se determinou o volume de água gasto no acionamento.

Os dados foram submetidos a tratamento estatístico através de análise de variância. Com base nos resultados obtidos nos ensaios fez-se uma avaliação no custo mensal e anual das despesas com água e 


\section{REA - Revista de estudos ambientais (Online) v.21, n. 2, p.48-57, jul./dez. 2019}

esgoto em um edifício multifamiliar com 12 andares, com 4 apartamentos por andar, considerando 4 habitantes por apartamento, os quais utilizam a descarga da bacia sanitária em média 5 vezes por dia. Para isso considerou-se as tarifas de serviços de água do município de Blumenau/SC conforme apresentados na Tabela 3.

Figura 4 - Variáveis estudadas no trabalho

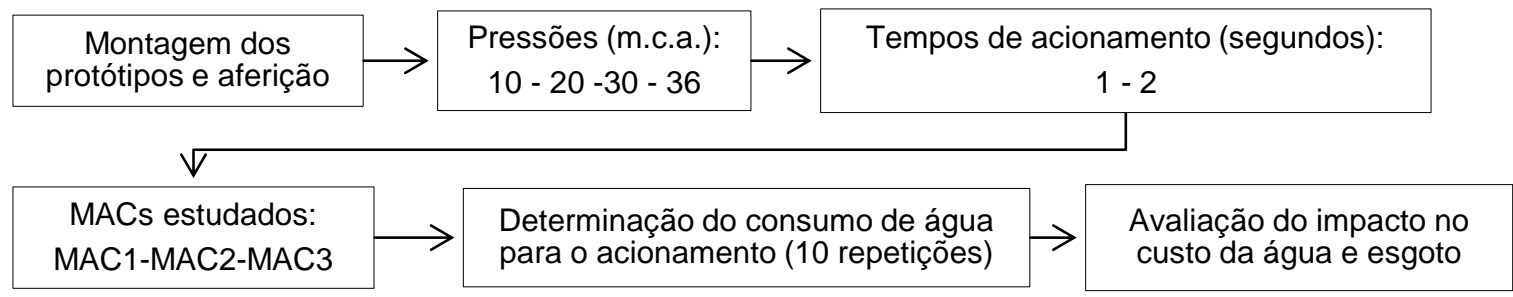

Fonte: Autores (2019)

Tabela 3 - Valores das tarifas de água e esgoto para o município de Blumenau/SC

\begin{tabular}{ccc}
\hline Categoria & Faixa de consumo $\left(\mathbf{m}^{3}\right)$ & Valores em R\$ \\
\hline Fornecimento de & Até 10 & 29,19 \\
água residencial & 11 a 30 & $+5,31 / \mathrm{m}^{3}$ \\
& 31 a 99.999 & $+6,77 / \mathrm{m}^{3}$ \\
\hline Coleta e tratamento & 0 a 10 & 33,46 \\
de esgoto & 11 a 30 & $+6,126 / \mathrm{m}^{3}$ \\
residencial & $>30$ & $+7,807 / \mathrm{m}^{3}$
\end{tabular}

Fonte: Adaptado SAMAE e BRK Ambiental (2018)

O cálculo da tarifa de resíduos sólidos é realizado através da equação 1 , sendo que - custo da coleta de resíduos sólidos é influenciado pelo consumo de água. Assim um maior ou menor consumo de água terá impacto neste custo também.

$$
\mathrm{TCL}=\mathrm{GL} * \mathrm{CT} * \mathrm{FU} * \mathrm{FF} * \mathrm{CA}
$$

Onde: $T C L$ - Taxa de coleta de lixo, em $R \$$; GL - Geração específica de lixo, igual a 0,00457; CT - Custo do tratamento por tonelada, $\mathrm{R} \$ 303,08$; FU - Fator de uso, em residência igual a $1 ; \mathrm{FF}-$ Fator de frequência (número de passadas do caminhão para a coleta na semana), até 3 coletas; CA Consumo médio faturado de água por economia, adotado critério 1. Critério 1 média anual do consumo faturado de água.

\section{Resultados}

Os volumes de água liberados pelos três MACs instalados junto à bacia sanitária com caixa acoplada são apresentados para um acionamento de 1 e 2 segundos com um volume de reservação inicial de $6 \mathrm{~L}$ de água (Tabela 4). Também são apresentadas a média das medições para cada pressão e o desvio padrão (S).

Com os dados apresentados na Tabela 4, verifica-se que as médias das amostras de água recolhidas durante o teste do MAC 1 variam entre 6,14 e $6,40 \mathrm{~L}$ para o tempo de acionamento de 1 segundo. Observa-se que quanto maior a pressão, maior o consumo de água. Ainda pode ser feita uma análise do desvio padrão, que para o MAC 1 varia entre 0,07 e 0,17 L. Os resultados para o desvio padrão apresentam baixa variação entre as amostras.

A quantidade de água consumida pelo MAC 1 em $2 \mathrm{~s}$ de acionamento apresentou valores médios variando de 6,12 a 6,46 L. Em relação ao ensaio anterior do mesmo MAC, houve pouca diferença, chegando a diminuir o consumo de água na pressão aplicada de 10 m.c.a. Nas demais pressões aplicadas no MAC 1 com $2 \mathrm{~s}$ de acionamento, houve aumento no consumo de água. A variação do desvio padrão foi pequena e muito satisfatória, ficando entre 0,04 e 0,12 . 
REA - Revista de estudos ambientais (Online)

v.21, n. 2, p.48-57, jul./dez. 2019

Tabela 4 - Consumos de água para os tempos de acionamento para os diferentes MACs e pressões impostas

\begin{tabular}{|c|c|c|c|c|c|c|c|c|c|c|c|c|c|c|}
\hline $\begin{array}{c}\text { Tempo } \\
(\mathbf{s})\end{array}$ & \multirow{5}{*}{$\begin{array}{c}\text { MAC } \\
\text { MAC } \\
1\end{array}$} & \multirow{2}{*}{$\begin{array}{c}\begin{array}{c}\text { Pressão } \\
\text { (m.c.a) }\end{array} \\
10\end{array}$} & \multicolumn{10}{|c|}{ Consumo de água (L) } & \multirow{2}{*}{\begin{tabular}{|l|} 
Méd \\
6,14
\end{tabular}} & \multirow{2}{*}{$\begin{array}{c}\begin{array}{c}\text { Desv. } \\
\text { Pad. }\end{array} \\
0,17\end{array}$} \\
\hline \multirow{12}{*}{1} & & & 6,55 & 6,00 & 6,30 & 6,00 & 6,00 & 6,20 & 6,10 & 6,10 & 6,00 & 6,10 & & \\
\hline & & 20 & 6,30 & 6,28 & 6,05 & 6,39 & 6,10 & 6,31 & 6,10 & 6,32 & 6,20 & 6,15 & 6,22 & 0,11 \\
\hline & & 30 & 6,30 & 6,40 & 6,40 & 6,20 & 6,42 & 6,30 & 6,40 & 6,41 & 6,40 & 6,29 & 6,35 & 0,07 \\
\hline & & 36 & 6,30 & 6,54 & 6,40 & 6,20 & 6,30 & 6,40 & 6,50 & 6,50 & 6,50 & 6,40 & 6,40 & 0,10 \\
\hline & \multirow{4}{*}{$\begin{array}{c}\text { MAC } \\
2\end{array}$} & 10 & 6,26 & 6,30 & 6,29 & 6,32 & 6,25 & 6,18 & 6,49 & 6,25 & 6,10 & 6,35 & 6,28 & 0,10 \\
\hline & & 20 & 6,50 & 6,38 & 6,35 & 6,10 & 6,40 & 6,20 & 6,38 & 6,40 & 6,25 & 6,25 & 6,32 & 0,11 \\
\hline & & 30 & 6,39 & 6,38 & 6,70 & 6,42 & 6,32 & 6,59 & 6,39 & 6,40 & 6,49 & 6,40 & 6,45 & 0,11 \\
\hline & & 36 & 6,60 & 6,52 & 6,52 & 6,61 & 6,42 & 6,62 & 6,49 & 6,70 & 6,60 & 6,52 & 6,56 & 0,08 \\
\hline & \multirow{4}{*}{$\begin{array}{c}\text { MAC } \\
3\end{array}$} & 10 & 6,65 & 6,54 & 6,70 & 6,40 & 6,70 & 6,67 & 6,43 & 6,69 & 6,55 & 6,40 & 6,57 & 0,12 \\
\hline & & 20 & 7,03 & 6,68 & 6,93 & 7,07 & 6,99 & 7,06 & 7,00 & 7,06 & 7,00 & 7,00 & 6,98 & 0,11 \\
\hline & & 30 & 7,35 & 7,10 & 7,14 & 7,32 & 7,09 & 7,38 & 7,38 & 7,41 & 7,00 & 7,22 & 7,24 & 0,14 \\
\hline & & 36 & 7,55 & 7,57 & 7,68 & 7,48 & 7,59 & 7,69 & 7,50 & 7,40 & 7,47 & 7,60 & 7,55 & 0,09 \\
\hline \multirow{12}{*}{2} & \multirow{4}{*}{$\begin{array}{c}\text { MAC } \\
1\end{array}$} & 10 & 6,08 & 6,09 & 6,13 & 6,10 & 6,07 & 6,14 & 6,10 & 6,19 & 6,19 & 6,13 & 6,12 & 0,04 \\
\hline & & 20 & 6,30 & 6,29 & 6,13 & 6,20 & 6,19 & 6,30 & 6,10 & 6,35 & 6,19 & 6,49 & 6,25 & 0,11 \\
\hline & & 30 & 6,59 & 6,26 & 6,42 & 6,48 & 6,40 & 6,39 & 6,20 & 6,38 & 6,30 & 6,45 & 6,39 & 0,11 \\
\hline & & 36 & 6,40 & 6,51 & 6,46 & 6,67 & 6,45 & 6,43 & 6,30 & 6,65 & 6,30 & 6,43 & 6,46 & 0,12 \\
\hline & \multirow{4}{*}{$\begin{array}{c}\text { MAC } \\
2\end{array}$} & 10 & 6,00 & 6,05 & 6,12 & 6,05 & 6,00 & 6,12 & 6,09 & 6,00 & 6,26 & 6,18 & 6,09 & 0,08 \\
\hline & & 20 & 6,11 & 6,42 & 6,35 & 6,08 & 6,30 & 6,22 & 6,30 & 6,24 & 6,30 & 6,18 & 6,25 & 0,10 \\
\hline & & 30 & 6,60 & 6,40 & 6,30 & 6,50 & 6,20 & 6,35 & 6,40 & 6,52 & 6,42 & 6,40 & 6,41 & 0,11 \\
\hline & & 36 & 6,42 & 6,28 & 6,62 & 6,42 & 6,39 & 6,40 & 6,61 & 6,51 & 6,43 & 6,40 & 6,45 & 0,10 \\
\hline & \multirow{4}{*}{$\begin{array}{c}\text { MAC } \\
3\end{array}$} & 10 & 6,60 & 6,82 & 6,66 & 6,60 & 6,63 & 6,60 & 6,60 & 6,77 & 6,59 & 6,60 & 6,65 & 0,08 \\
\hline & & 20 & 7,20 & 7,14 & 7,19 & 7,07 & 7,11 & 7,20 & 7,23 & 7,40 & 7,14 & 7,60 & 7,23 & 0,15 \\
\hline & & 30 & 7,42 & 7,40 & 7,30 & 7,40 & 7,18 & 7,25 & 7,37 & 7,20 & 7,18 & 7,37 & 7,31 & 0,09 \\
\hline & & 36 & 7,59 & 7,42 & 7,35 & 7,50 & 7,36 & 7,70 & 7,57 & 7,55 & 7,49 & 7,51 & 7,50 & 0,10 \\
\hline
\end{tabular}

Onde: Méd = média; Desv. Pad. = desvio padrão; MAC = Mecanismo de acionamento; s = segundos; m.c.a. = metros de coluna de água

Fonte: Autores (2019)

Fazendo uma análise dos resultados obtidos durante o ensaio do MAC 2, observase que também ocorreram variações nos resultados coletados e um maior consumo de água por parte da bacia sanitária com caixa acoplada conforme a pressão aumentou. Pode-se observar ainda que o desvio padrão, calculado para o MAC 2 com tempo de acionamento de $1 \mathrm{~s}$ varia entre 0,08 e $0,11 \mathrm{~L}$, ou seja, pouca variação entre as medições.

Nos acionamentos de $2 \mathrm{~s}$ o MAC 2 teve um consumo médio bem variado, entre 6,09 e $6,45 \mathrm{~L}$, um consumo relativamente razoável e de acordo com a NBR 15491 (ABNT, 2010), que permite um volume de descarga reduzido de 6,8 L. Em comparação ao ensaio com o mesmo MAC para um tempo menor, o consumo de água para um acionamento de $2 \mathrm{~s}$ foi reduzido consideravelmente em todas as pressões aplicadas. O desvio padrão do MAC 2 ficou entre 0,08 e $0,11 \mathrm{~L}$ para o tempo de $1 \mathrm{~s}$, ou seja, variações muito pequenas, indicando que as medições possuem boa homogeneidade.

O MAC 3 apresentou resultados bastante variados e com um consumo de água elevado quando comparado aos demais MACs. Observa-se que os valores de consumo do MAC 3 são altos, chegando a uma média de $7,55 \mathrm{~L}$ de água por segundo para uma pressão de 36 m.c.a., representando $1,55 \mathrm{~L}$ a mais do que a reservação inicial. A variação da média das medições, entre as pressões de 10 e 36 m.c.a. foi de 0,98 L. A quantidade de água consumida pelo MAC 3 em algumas pressões ultrapassa o valor de VDR estabelecido pela 


\section{REA - Revista de estudos ambientais (Online) v.21, n. 2, p.48-57, jul./dez. 2019}

norma de 6,8 L. O desvio padrão varia de 0,09 a $0,15 \mathrm{~L}$, também mostrando a homogeneidade entre as medições realizadas.

O MAC 3 ultrapassou o limite do volume da caixa de descarga de $6,8 \mathrm{~L}$ nas pressões de 20, 30 e 36 m.c.a., chegando em um valor médio de consumo de $7,5 \mathrm{~L}$ em $2 \mathrm{~s}$ de acionamento. Os consumos de água pelo MAC $3 \mathrm{em} 2 \mathrm{~s}$ de acionamento, foram um pouco maiores em relação ao mesmo MAC em um tempo menor, exceto na pressão aplicada de 36 m.c.a., em que o valor médio foi um pouco menor. $O$ desvio padrão das amostras coletadas durante o ensaio do MAC 3 ficou entre 0,08 e 0,16 , uma variação considerada pequena.

$\mathrm{Na}$ Tabela 5 é apresentada uma análise de variância ficando claro que o tipo de MAC e a pressão da rede são significativos na vazão da caixa acoplada. Já o tempo de acionamento não se mostrou significativo. O nível de confiança da análise foi adotado como $95 \%$. Todas as interações entre as variáveis também se mostraram significativas na análise de variância.

A Figura 5 apresenta o consumo médio de água para as diferentes condições de ensaio, constatando-se que o consumo de água cresce em função do aumento da pressão da rede para os três MAC estudados. Também é evidenciado o maior consumo do MAC 3 em relação aos demais MAC e em relação à faixa de 6 a 6,8 L estabelecida por norma. $\mathrm{Na}$ Figura 5 também são apresentadas linhas de tendência do consumo de água para os diferentes MACs, com tempos de acionamento frente às diferentes pressões.

Tabela 5 - Análise de variância das médias de consumo de água para os diferentes tipos de MACs, tempo de acionamento e pressão

\begin{tabular}{cccccccc} 
Fonte & SQ & GDL & MQ & Teste F & F Tab & Prob. & SIG \\
\hline A: MAC & 34,97 & 2 & 17,48 & 1379,60 & 3,04 & $0 \%$ & sim \\
B: Tempo & 0,00 & 1 & 0,00057 & 0,05 & 3,88 & $83 \%$ & não \\
C: Pressão & 8,75 & 3 & 2,92 & 230,27 & 2,65 & $0 \%$ & sim \\
AB & 0,37 & 2 & 0,19 & 14,72 & 3,04 & $0 \%$ & sim \\
AC & 2,60 & 6 & 0,43 & 34,15 & 2,14 & $0 \%$ & sim \\
BC & 0,13 & 3 & 0,04 & 3,32 & 2,65 & $2 \%$ & sim \\
ABC & 0,17 & 6 & 0,03 & 2,29 & 2,14 & $4 \%$ & sim \\
Erro & 2,74 & 216 & 0,01 & & & & \\
\hline Total & 49,73 & 239 & & & & & \\
\hline
\end{tabular}

Onde: $\mathrm{SQ}=$ soma quadrada; $\mathrm{GDL}=$ graus de liberdade; $\mathrm{MQ}=$ média quadrada; $\mathrm{F}$ = Fischer; $\mathrm{F}$ Tab = Fischer tabelado; Prob. $=$ probabilidade $;$ SIG = significativo .

Fonte: Autores (2019)

\section{Figura 5 - Consumo de água em função da pressão, do tempo de acionamento e do MAC da bacia sanitária}

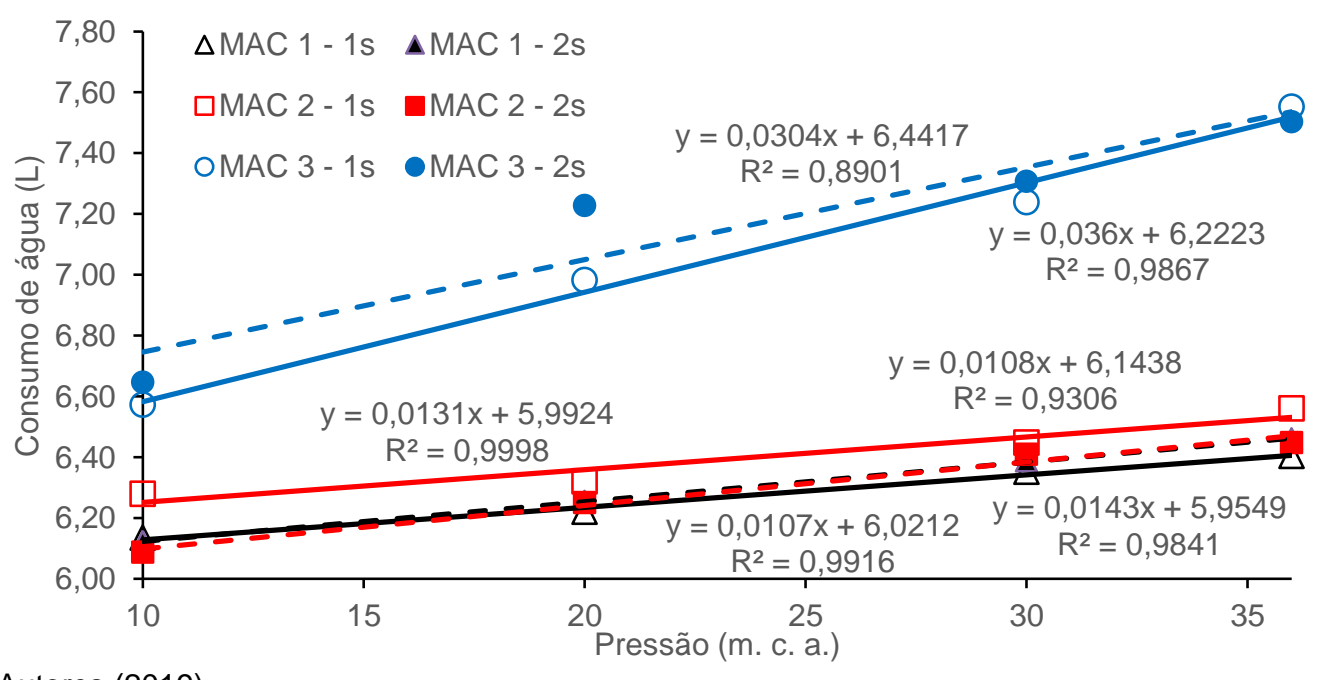

Fonte: Autores (2019) 


\section{REA - Revista de estudos ambientais (Online) v.21, n. 2, p.48-57, jul./dez. 2019}

Realizou-se, também, uma avaliação do impacto que $o$ aumento na vazão causaria no custo do consumo de água e no custo da coleta do esgoto de um edifício multifamiliar para o aumento do consumo de água além do volume de reservação inicial de $6 \mathrm{~L}$ em um edifício de 12 pavimentos (Tabela 6). Considerou-se as diferentes pressões (em função da altura do edifício) e tempos de acionamento.

Tabela 61 - Acréscimo no consumo de água de descarga da bacia sanitária em um edifício de 12 pavimentos

\begin{tabular}{c|c|c|c|c}
\hline $\begin{array}{c}\text { Tempo } \\
(\mathbf{s})\end{array}$ & MAC & $\begin{array}{c}\text { Acréscimo médio } \\
\text { por descarga (L) }\end{array}$ & $\begin{array}{c}\text { Acréscimo no consumo } \\
\text { mensal }\left(\mathbf{m}^{\mathbf{3}}\right)\end{array}$ & $\begin{array}{c}\text { Acréscimo no } \\
\text { consumo anual }\left(\mathbf{m}^{\mathbf{3}}\right)\end{array}$ \\
\hline \multirow{3}{*}{1} & 1 & 0,24 & 6,93 & 84,29 \\
\cline { 2 - 5 } & 2 & 0,26 & 7,52 & 91,44 \\
\cline { 2 - 5 } & 3 & 0,96 & 27,66 & 336,49 \\
\hline \multirow{3}{*}{2} & 1 & 0,25 & 7,14 & 86,92 \\
\cline { 2 - 5 } & 2 & 0,37 & 10,52 & 127,97 \\
\cline { 2 - 5 } & 3 & 1,06 & 30,67 & 373,14 \\
\hline
\end{tabular}

Fonte: Autores (2019)

De acordo com Duarte (2017), em
Santa Catarina cada pessoa gasta
diariamente $154 \mathrm{~L}$ de água. Considerando
este consumo e as diferentes tarifas de
consumo de água e esgoto apresentadas na
Tabela 3 determinou-se 0 impacto do acréscimo no consumo de água de descarga no edifício estudado para cada MAC e tempo de acionamento (Tabela 7).

Tabela 7 - Acréscimo do custo de água e esgoto em função do tipo do MAC de descarga e tempo de acionamento em um edifício de 12 pavimentos

\begin{tabular}{c|c|c|c|c}
\hline $\begin{array}{c}\text { Tempo } \\
(\mathbf{s})\end{array}$ & MAC & $\begin{array}{c}\text { Acréscimo mensal } \\
\text { do custo } \mathbf{( R \mathbf { ~ } )}\end{array}$ & $\begin{array}{c}\text { Acréscimo anual } \\
\text { do custo (R\$) }\end{array}$ & $\begin{array}{c}\text { Representatividade } \\
\text { no custo total (\%) }\end{array}$ \\
\hline \multirow{3}{*}{1} & 1 & 100,99 & $1.228,68$ & 0,78 \\
\cline { 2 - 5 } & 2 & 109,55 & $1.332,88$ & 0,84 \\
\cline { 2 - 5 } & 3 & 403,15 & $4.905,00$ & 3,12 \\
\hline \multirow{3}{*}{2} & 1 & 104,14 & $1.266,99$ & 0,80 \\
\cline { 2 - 5 } & 2 & 153,32 & $1.865,36$ & 1,19 \\
\cline { 2 - 5 } & 3 & 447,06 & $5.439,28$ & 3,46 \\
\hline
\end{tabular}

Fonte: Autores (2019)

Observa-se que com o aumento do tempo de acionamento e do tipo do MAC houve aumento nos valores consumidos de água (Tabela 7). O MAC 3 foi o que causou maior aumento no consumo de água, causando uma diferença mensal no valor a pagar de $\mathrm{R} \$ 447,06$ para o tempo de acionamento de $2 \mathrm{~s}$. Anualmente, o custo pode ter um aumento de até $\mathrm{R} \$ 5.439,28$.

\section{Conclusões}

Os resultados obtidos neste trabalho permitiram comprovar que o aumento da pressão em edifícios de múltiplos pavimentos, provoca um maior consumo de água junto às bacias sanitárias com caixa acoplada, também associado ao tipo de MACs de reparo instalados.

Foi possível constatar a correlação entre o aumento da pressão com o consumo mais elevado de água. O MAC 3 foi o que mais demandou água, chegando para 1 segundo de acionamento em um consumo de $7,55 \mathrm{~L}$ de água e em $2 \mathrm{~s}$ de acionamento 7,50 $\mathrm{L}$, ambos para uma pressão de 36 m.c.a. Estes valores ultrapassam 0 valor estabelecido pela NBR 15491 (ABNT, 2010), de que uma bacia sanitária com volume reduzido não deve consumir mais de $6,8 \mathrm{~L}$ de água por acionamento.

O MAC 1 é o que menos demandou água, em média 6,40 L de água por descarga em 1 segundo de acionamento quando se utiliza uma pressão de 36 m.c.a. Já em 2 s de 


\title{
REA - Revista de estudos ambientais (Online) v.21, n. 2, p.48-57, jul./dez. 2019
}

acionamento, o MAC 2 possui uma demanda média de $6,45 \mathrm{~L}$ de água por descarga.

Com os valores de consumo de água coletados durante os ensaios, pôde-se fazer uma análise do impacto econômico, causado pelo consumo de água nos MACs. Para um edifício residencial com 12 pavimentos, o impacto econômico gerado por esse acréscimo no consumo de água, implicou em um aumento no custo anual de água, esgoto e taxa de lixo em até $R \$ 5.439,28$.

\section{Evaluation of Water Demand Due to Different Pressures in Sanitary Basins with Box Coupled in Residential Buildings}

\begin{abstract}
The study of pressure in repair MACs for sanitary basins can provide a considerable reduction in the consumption of water in residential units, making the system more economical and sustainable. The sanitary basins are one of the devices responsible for the highest consumption of water and the high pressure is an aggravating factor. This consumption is due to a loss of water that can occur when the box is refilled, while the push button is being pressed. The present study aimed to study the water consumption in sanitary basins with coupled box, using three types of MAC models for repair that were submitted to different pressures. The discharge was activated for two times, 1 and $2 \mathrm{~s}$, and for each time four different pressure values were adopted, 10, 20, 30 and 36 MWC, repeating the procedure ten times for each combination and for each MAC. MAC 3 was the one with the highest average variation in water consumption, which was between $6.57 \mathrm{~L}$ and $7.55 \mathrm{~L}$. The increase in water consumption by MAC3 generated an economic impact of $R \$ 5.439,28$ on the annual water and sewage bill in a multifamily building, with 48 apartments spread over 12 floors.
\end{abstract}

Keywords: Sanitary basin; Coupled box; Water demand; Waste.

\section{Referências}

ANNECCHINI, K. P. V. Aproveitamento da água da chuva para fins não potáveis na cidade de Vitória (ES). 2005. 150 f. Dissertação (Mestrado) Curso de Engenharia Ambiental, Tecnológico, Universidade Federal do Espírito Santo, Vitória, 2005.

ASSOCIAÇÃO BRASILEIRA DE NORMAS TÉCNICAS. NBR 15.097-1: Aparelhos sanitários de material cerâmico Parte 1: Requisitos e métodos de ensaio. 2 ed. Rio de Janeiro, 2017. 63 p.

ASSOCIAÇÃO BRASILEIRA DE NORMAS TÉCNICAS. NBR 15491: Caixa de descarga para limpeza de bacias sanitárias: Requisitos e métodos de ensaio. Rio de Janeiro, 2010. 40 p.

BRK AMBIENTAL. Estrutura tarifária. 2017. Disponível em: https://www.brkambiental.com.br/blumenau/aguae-esgoto/sua-conta/estrutura-tarifaria/\#tabe-id-53. Acesso em: 13 mar. 2018.

CABRAL, J. J. S. P.; AGUIAR, S. R.; OLIVEIRA, M. A.; MONTENEGRO, S. M. G. L. Projeto piloto de uma caixa de descarga eficiente, econômica e à prova de vazamentos. 2016. Disponível em: http://www.scielo.br/pdf/esa/2017nahead/18094457-esa-s1413-41522017127339.pdf. Acesso em: 15 jan. 2019.

COELHO, M. G. Avaliação dos usos finais de água em residências unifamiliares localizadas em Blumenau - SC. Relatório de Iniciação Científica, Universidade Federal de Santa Catarina, 2008. 73p.

DUARTE, G. O consumo diário de água em Santa Catarina é maior do que o recomendado pela ONU. Diário Catarinense, Florianópolis, 22 mar. $2017 . \quad$ Disponível em: http://dc.clicrbs.com.br/sc/estilo-devida/noticia/2017/03/consumo-diario-de-agua-emsanta-catarina-e-maior-do-que-o-recomendadopela-onu-9753926.html. Acesso em:15 jan. 2019.

FABRICANTE 1. Caixa acoplada. 2018, Disponível em: https://www.blukit.com.br/produto/detalhe/kitcompleto-universal-para-caixas-acopladas-commecanismo-acionador-superior. Acesso em: 15 jan. 2019.

FABRICANTE 2. Caixa acoplada. 2018, Disponível em: http://censi.com.br/produto/detalhe/mecanismode-saida-para-caixas-acopladas-4. Acesso em: 15 jan. 2019.

\section{FABRICANTE 3. Caixa acoplada. 2018, Disponível 3. Caixa acoplada. $\begin{array}{r}2018 \\ \text { em: }\end{array}$ https://www.deca.com.br/produto/kit-universal- caixa-de-descarga-simples-nao-aplicavel- 1100si5401/. Acesso em: 15 jan. 2019.}

FRACASSO, U. R. Análise de custos na implantação de um sistema de reaproveitamento de águas pluviais em uma residência unifamiliar. 2017. 43 f. (Monografia). 
REA - Revista de estudos ambientais (Online) v.21, n. 2, p.48-57, jul./dez. 2019

Engenharia Civil. Universidade do Planalto Catarinense, Lages, 2017.

LOMBARDI, L. R. Dispositivos poupadores de água em um sistema predial: Análise da viabilidade técnico - econômica de implementação no instituto de pesquisas hidráulicas. 2012. 77 f. (Graduação de Engenharia Civil). Departamento de Engenharia Civil, Universidade Federal do Rio Grande do Sul, Porto Alegre, 2012. Disponível em: https://www.lume.ufrgs.br/bitstream/handle/10183/ 79746/000897368. pdf? sequence $=1$ \&isAllowed=y. Acesso em: 15 jan. 2019.

OLIVEIRA, S. M. Aproveitamento da água da chuva e reuso de água em residências unifamiliares: estudo de caso em Palhoça - SC. 2005. 149 f. TCC (Graduação) - Curso de Engenharia Civil, Universidade Federal de Santa Catarina, Florianópolis, 2005.

REALI, M. A. P.; MORUZZI, R. B.; ENGA, A. P. P.; CARVALHO, K. Q. Instalações prediais de água fria. 2002. Disponível em: https://organizacaotc.files.wordpress.com/2014/04 /c3a1gua-fria-manual-de-instalac3a7c3b5esprediais.pdf. Acesso em: 15 jan. 2019.

SERVIÇO MUNICIPAL DE ÁGUA E ESGOTO SAMAE. Reajuste dos serviços de abastecimento de água. 2017. Disponível em: http://samae.com.br/pagina/123_Reajuste-dosservicos-de-abastecimento-de-agua---2015.html. Acesso em: 1 nov. 2017.

TOMAZ, P. Previsão de consumo de água não potável. In.: TOMAZ, P. Interface das instalações prediais de água e esgoto com os serviços públicos. Guarulhos/SP:PTE, 2009, p.12-83.

UCHIDA, C.; OLIVEIRA, L. H. As bacias sanitárias com sistema de descarga dual e a redução do consumo de água em edifício residencial multifamiliar. 2006. Disponível em:http://www.infohab.org.br/entac2014/2006/arti gos/ENTAC2006_3450_3459.pdf. Acesso em: 15 jan. 2019. 